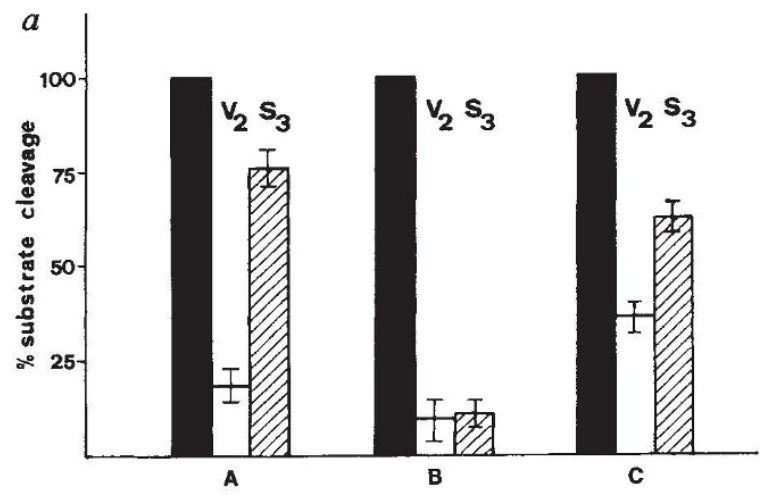

Botulinum toxin

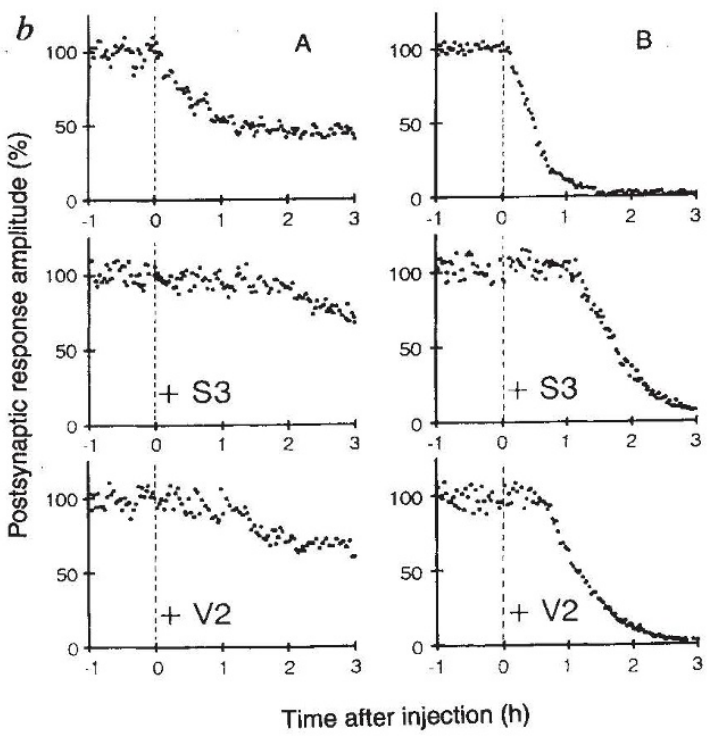

FIG. 2 The proteolytic activity (a) and blockade of neuroexocytosis in Aplysia neurons (b) of botulinum neurotoxin A, B and $\mathrm{C}$ are inhibited by VAMP- and SNAP-25-derived peptides. a, Proteolytic activities of botulinum A (left), B (centre) and C (right), measured as before ${ }^{7}$, in the absence of added peptides, were taken as $100 \%$ (full bars) and compared with those found in the presence of $500 \mu \mathrm{M}$ V2 or S3 (open and hatched bars, respectively). $b$, Acetylcholine release of neurons of the buccal ganglion of $A$. californica was assayed as before $^{7}$. Neurons were injected with botulinum A (left panels) or B (right panels), either alone (upper panels) or in neurons preinjected with S3 (middle row) or V2 (bottom panels). V2 and $\mathrm{S} 3$ alone did not affect acetylcholine release.

californica. V2 and S3 do not affect neuroexocytosis, but inhibit the blockade of acetylcholine release induced by botulinum $\mathrm{A}$ and $\mathrm{B}$; $\mathrm{C}$ has little effect. Scrambled peptides have no effect on neurotransmitter release and on neuro-

1. Montecucco, C. \& Schiavo, G. Molec. Microbiol. 13, 1-8 (1994).

2. Huttner, W. Nature 32, 3-4 (1993)

3. Ferro-Novick, S. \& Jahn, R. Nature 370, 191-193 (1994)

. Sölnner, T. et al. Nature 362, 318-324 (1993).

5. Rothman, J. \& Warren, G. B. Curr. Biol. 4, 220-233 (1994).

6. Bennett, M. K. \& Scheller, R. H. A. Rev. Biochem. 63 63-100 (1994).

7. Schiavo, G. et al. Nature 359, 832-835 (1992).

8. Shone, C. C. et al. Eur. J. Biochem. 217, 965-971 (1993).

9 Yamasaki, S. et al. J. biol. Chem. 269, 12764-12772 (1994).

10 Binz, T. et al. J. biol. Chem. 269, 1617-1620 (1994). $11 \mathrm{Vu}, \mathrm{T}$. H. et al. Nature 353, 674-677 (1991). toxin action. The neurotoxin recognition motif has to be exposed to the surface and, if it acts as an epitope, antibodies against the SNARE motif should recognize all three targets. Immunoblotting with affinity-purified polyclonal antibodies anti-V2 and anti-S3 recognize VAMP, SNAP-25 and syntaxin at the same time. Patterns of crossreactivity are also found with some polyclonal antibodies raised against recombinant SNAREs. Parallel to this antibody crossreactivity, one would expect a cross-recognition of the targets by the various neurotoxins. Indeed, botulinum $\mathrm{A}$ does not cleave VAMP, but inhibits VAMP proteolysis of botulinum $\mathrm{B}$ and tetanus neurotoxins. Conversely, tetanus neurotoxins and botulinum B neurotoxins inhibit the botulinum A cleavage of SNAP-25.

On the basis of these findings, we suggest that tetanus and botulinum toxins recognize their substrates via a double interaction with region $\mathbf{A}$, a structural motif common to the three targets, and region $\mathbf{B}$, the segment containing the peptide bond to be cleaved. $\mathbf{B}$ differs among the different neurotoxins and is located at variable distance from $\mathbf{A}$ in the primary structure of the three targets (Fig. 1). Available data ${ }^{1,7-10}$ indicate that region $\mathbf{A}$ is always aminoterminal to $\mathbf{B}$. The present identification of a recognition site A distinct from the cleavage site B explains recent analysis of the minimal substrate segment still cleaved by the toxins $^{8-10}$, although the relative contribution of $\mathbf{A}$ and $\mathbf{B}$ to specificity of binding and rate of proteolysis remains to be studied. The negative charges of the motif appear essential.

This report is, to our knowledge, the first example of a double recognition of a substrate by a metalloproteinase and parallels that of the serine-protease thrombin versus the thrombin receptor ${ }^{11}$. It suggests a new method for the design of peptide inhibitors of clostridial neurotoxins of potential therapeutic value.

VAMP, SNAP-25 and syntaxin are essential to the process of vesicle docking-priming-fusion with the target mem- brane ${ }^{1-4}$. They possess multiple copies of a motif, not shared by any known neuronal protein. Among eukaryotic proteins, the motif is found also in yeast BPP4 and amino-end-recognizing proteins, as deduced from an inspection of the Swiss Protein databank with the FASTA program. This result suggests that VAMP, SNAP-25 and syntaxin are the sole neuronal targets of the proteolytic activity of clostridial neurotoxins. Moreover, it emphasizes the functional importance of this motif as a site that cannot be mutated under the selective pressure of neurotoxin action without loss of function. The physiological function of this segment is unknown, but a mutational scanning of the motif via site-directed mutagenesis and cell transfection will allow evaluation of its role in the exo-endocytosis cycle.

Ornella Rossetto, Giampietro Schiavo Cesare Montecucco*

Dipartimento di Scienze Biomediche and Centro CNR Biomembrane,

Università di Padova,

75 Via Trieste, 75 - 35121 Padova, Italy

Bernard Poulain, Florence Deloye

Laboratoire de Neurobiologie Moléculaire et Cellulaire du CNRS,

91118 Gif-sur-Yvette, France

Luisa Lozzi

Dipartimento di Biologia Molecolare,

Università di Siena,

Le Scotte, 53100 Siena, Italy

Clifford C. Shone

Protein Toxins Section, CAMR,

Porton Down, Salisbury SP4 OJG, UK

*Author for correspondence.

\section{Visual reference}

SIR - It has been drawn to our attention that a paper written by Redies and others ${ }^{1}$ on neuronal responses to subjective contours in the visual cortex of cats was not cited in our paper published last year ${ }^{2}$ in which we suggested a new view of the primary visual cortex of macaque monkeys as an image-processing area. We did refer Redies et al. ${ }^{1}$ in an interview for Science published in 1992, but in ref. 2 we cited only those papers that we felt were directly relevant to the points addressed therein. Nevertheless, we refer readers interested in the neurophysiology of subjective contours to the work of Redies et al.

David Grosof

Department of Ophthalmology,

Washington University, School of Medicine, St Louis, Missouri 63110, USA

Michael Hawken

Robert Shapley

Center for Neural Science,

New York University,

New York 10003-6621, USA

1. Redies, C., Crook, J. M. \& Creutzfeldt, O. D. Expl Brain Res. 61, 469-481 (1986).

2. Grosof, D. H., Shapley, R. M. \& Hawken, M. J. Nature 365, 550-552 (1993).

3. Winckelgren, I. Science 256, 1520-1521 (1992). 